\title{
Polarized Protons in the Fermilab Main Injector
}

\author{
W. Lorenzon*t and C.A. Aidala \\ Randall Laboratory of Physics, University of Michigan, Ann Arbor, Michigan 48109-1040, USA \\ E-mail: lorenzoneumich.edu
}

The Main Injector at Fermilab currently produces high-intensity beams of protons at energies up to $120 \mathrm{GeV}$ for a variety of physics experiments. Polarizing the protons in the Main Injector will open up opportunities for a rich spin physics program at Fermilab. To achieve polarized proton beams in the Main Injector accelerator complex, detailed design and costing studies using a novel single Siberian snake approach are needed. In particular, detailed spin tracking simulations with realistic parameters based on the existing facility will be required to demonstrate that the present conceptual design can preserve beam polarization throughout the acceleration chain. The spin tracking further needs to investigate the tolerance of various machine and beam errors for preserving beam polarization. We report on the motivation, the present status and plans to develop polarized beams at Fermilab.

$X V^{\text {th }}$ International Workshop on Polarized Sources, Targets, and Polarimetry,

September 9-13, 2013

Charlottesville, Virginia, USA

\footnotetext{
*Speaker.

$\dagger$ representing the E1027 collaboration
} 


\section{Polarized Drell-Yan}

Polarized Drell-Yan scattering has become a major milestone in the hadronic physics community, motivated by a fundamental prediction of QCD that postulates that a (naïvely) time-reversal-odd distribution function, such as the Sivers function [1] measured in Drell-Yan must change sign [2, 3] if measured in semi-inclusive deep inelastic scattering (SIDIS). The Sivers function is described by a transverse-momentum dependent distribution function that captures non-perturbative spin-orbit effects inside a polarized proton. The experimental verification of the sign change goes to the heart of the gauge formulation of QCD and would be crucial to confirm the validity of our present conceptual framework for analyzing hard hadronic reactions.

The HERMES experiment [4] at DESY and the COMPASS experiment [5] at CERN have measured single transverse spin asymmetries to high precision, and global fits to the Sivers function have been performed [6]. In order to make a meaningful comparison of sign and shape, comparable measurements are needed for single spin asymmetries in the Drell-Yan process. While there are plans for a wide variety of experiments around the globe that aim to measure polarized Drell-Yan either with a polarized beam or a polarized target (see Table 1), none of them is optimized for DrellYan, except for the SeaQuest dimuon spectrometer at the Fermilab Main Injector. At present, only the COMPASS experiment is scheduled to run in the near future. COMPASS is scheduled to take data in 2014 (and again in 2018) for one year and expects to measure the sign of the Sivers function in the same kinematics as semi-inclusive DIS with a statistical precision of $\delta A_{N} / A_{N}$ of $1 \%-2 \%$ (in a single $x_{f}$-bin centered at $x_{f}=0.2$ ). A polarized Drell-Yan experiment such as E1027/polDY using the SeaQuest spectrometer is needed, however, to measure the sign, the magnitude, and possibly the shape of the Sivers function with sub-\% precision over several $x_{f}$-bins (see Fig. 1).

\begin{tabular}{|c|c|c|c|c|c|}
\hline experiment & particles & energy & $x_{1}$ or $x_{2}$ & luminosity & timeline \\
\hline $\begin{array}{l}\text { COMPASS } \\
(\mathrm{CERN})\end{array}$ & $\pi^{ \pm}+p^{\uparrow}$ & $\begin{array}{l}160 \mathrm{GeV} \\
\sqrt{s}=17.4 \mathrm{GeV}\end{array}$ & $x_{2}=0.2-0.3$ & $1 \times 10^{32} \mathrm{~cm}^{-2} \mathrm{~s}^{-1}$ & $\begin{array}{r}2014, \\
2018\end{array}$ \\
\hline $\begin{array}{l}\text { PAX } \\
\text { (GSI) }\end{array}$ & $p^{\uparrow}+\bar{p}$ & $\begin{array}{l}\text { collider } \\
\sqrt{s}=14 \mathrm{GeV}\end{array}$ & $x_{1}=0.1-0.9$ & $2 \times 10^{30} \mathrm{~cm}^{-2} \mathrm{~s}^{-1}$ & $>2017$ \\
\hline $\begin{array}{l}\text { PANDA } \\
\text { (GSI) }\end{array}$ & $\bar{p}+p^{\uparrow}$ & $\begin{array}{l}15 \mathrm{GeV} \\
\sqrt{s}=5.5 \mathrm{GeV}\end{array}$ & $x_{2}=0.2-0.4$ & $2 \times 10^{32} \mathrm{~cm}^{-2} \mathrm{~s}^{-1}$ & $>2016$ \\
\hline $\begin{array}{l}\text { NICA } \\
\text { (JINR) }\end{array}$ & $p^{\uparrow}+p$ & $\begin{array}{l}\text { collider } \\
\sqrt{s}=20 \mathrm{GeV}\end{array}$ & $x_{1}=0.1-0.8$ & $1 \times 10^{30} \mathrm{~cm}^{-2} \mathrm{~s}^{-1}$ & $>2014$ \\
\hline $\begin{array}{l}\text { PHENIX } \\
\text { (RHIC) }\end{array}$ & $p^{\uparrow}+p$ & $\begin{array}{l}\text { collider } \\
\sqrt{s}=200 \mathrm{GeV}\end{array}$ & $x_{1}=0.05-0.1$ & $2 \times 10^{32} \mathrm{~cm}^{-2} \mathrm{~s}^{-1}$ & $>2018$ \\
\hline $\begin{array}{l}\text { SeaQuest } \\
\text { (FNAL E906) }\end{array}$ & $p+p$ & $\begin{array}{l}120 \mathrm{GeV} \\
\sqrt{s}=15 \mathrm{GeV}\end{array}$ & $\begin{array}{l}x_{1}=0.3-0.9 \\
x_{2}=0.1-0.45\end{array}$ & $3.4 \times 10^{35} \mathrm{~cm}^{-2} \mathrm{~s}^{-1}$ & $\begin{array}{r}2013- \\
2015 \\
\end{array}$ \\
\hline $\begin{array}{l}\text { polDY } \\
\text { (FNAL E1027) }\end{array}$ & $p^{\uparrow}+p$ & $\begin{array}{l}120 \mathrm{GeV} \\
\sqrt{s}=15 \mathrm{GeV}\end{array}$ & $x_{1}=0.3-0.9$ & $2 \times 10^{35} \mathrm{~cm}^{-2} \mathrm{~s}^{-1}$ & $>2016$ \\
\hline
\end{tabular}

Table 1: Planned polarized Drell-Yan experiments. $x_{1}$ and $x_{2}$ are the parton momentum fractions in the beam and target, respectively. Note that the polDY luminosity of $2 \times 10^{35} \mathrm{~cm}^{-2} \mathrm{~s}^{-1}$ can be achieved with only $10 \%$ of the available beam time at the Main Injector. The target-heating-limited luminosity is in fact $1 \times 10^{36} \mathrm{~cm}^{-2} \mathrm{~s}^{-1}$. 


\subsection{Opportunities at Fermilab}

There is a great opportunity at Fermilab, at a relatively modest cost $(\$ 10 \mathrm{M})$ and with minimal impact on the neutrino program (10\% of beam time) to set up the best polarized Drell-Yan experiment to perform this fundamental test of the gauge structure of QCD (see Fig. 1). It could be the beginning of a world-class polarized proton program [7, 8] at Fermilab that is complementary to the spin physics programs at RHIC and Jefferson Lab. The big attraction for a polarized DrellYan program at the Fermilab Main Injector is the high luminosity in combination with an existing hydrogen target and a spectrometer that are well-understood, fully functioning, and optimized for Drell-Yan at the end of data collection for the E906/SeaQuest experiment (estimated to be in late 2015). Furthermore, the E906/SeaQuest spectrometer accommodates a large coverage in parton momentum fraction $x$, i.e. $x_{1}=0.35-0.85$ covering the valence quark region, and $x_{2}=0.1-0.45$ covering the sea quark region. Although the Sivers function can be measured for both the valence quarks or the sea quarks, the SIDIS data from HERMES and COMPASS constrain the valence quark Sivers function much more than the sea quark Sivers function [6, 9]. Thus, using a polarized beam promises to be a substantial advantage over a polarized target.

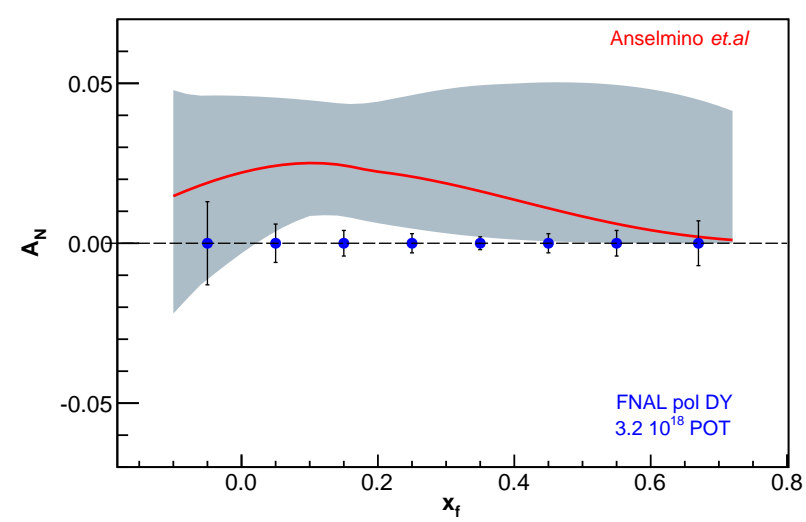

Figure 1. Single spin asymmetry $A_{N}$ as a function of $x_{f}$. The expected statistical uncertainties (blue solid circles) for a $70 \%$ polarized beam on an unpolarized target and $3.2 \times 10^{18}$ protons on target are (arbitrarily) plotted on the zeroline. The red line indicates the prediction for the Sivers SSA [10] based on the SIDIS results of HERMES and COMPASS, and the gray shaded area represents the $\sqrt{20}$-sigma error band.

\section{Polarization Scheme}

To accelerate polarized protons in the Fermilab Main Injector, modifications are needed [8] in most accelerator stages, as shown in Fig. 2. The major new components needed are a polarized ion source, and a superconducting Siberian snake in the Main Injector.

The Spin@Fermi collaboration has estimated [8] that an ion source that produces $1 \mathrm{~mA}$ at the source can deliver up to $150 \mathrm{nA}\left(9.5 \times 10^{11} \mathrm{p} / \mathrm{s}\right)$ average beam current to the experiment, using 30 two-second cycles and slip stacking in the Main Injector. Since the liquid targets are designed for average beam currents of about $80 \mathrm{nA}$, i.e. three times the beam current foreseen for SeaQuest, a luminosity of $1 \times 10^{36} \mathrm{~cm}^{-2} \mathrm{~s}^{-1}$ could be obtained if $50 \%$ of the total beam time was allocated to the experiment. However, a polarized Drell-Yan program at Fermilab is only feasible if not more than $10 \%$ of the available beam time is diverted from the neutrino program. Thus, assuming $10 \%$ of the available beam time was allocated to the experiment, which corresponds to a luminosity of $2.0 \times 10^{35} \mathrm{~cm}^{-2} \mathrm{~s}^{-1}$, and further assuming a beam polarization of $70 \%$, a running efficiency of $50 \%$ and a running period of 2 years, not only the sign, but also the size and shape of the Sivers function can be measured. This is illustrated in Fig. 1, where the red line indicates the prediction for the Sivers asymmetry, and the gray shaded area represents the $\sqrt{20}$-sigma error band. 


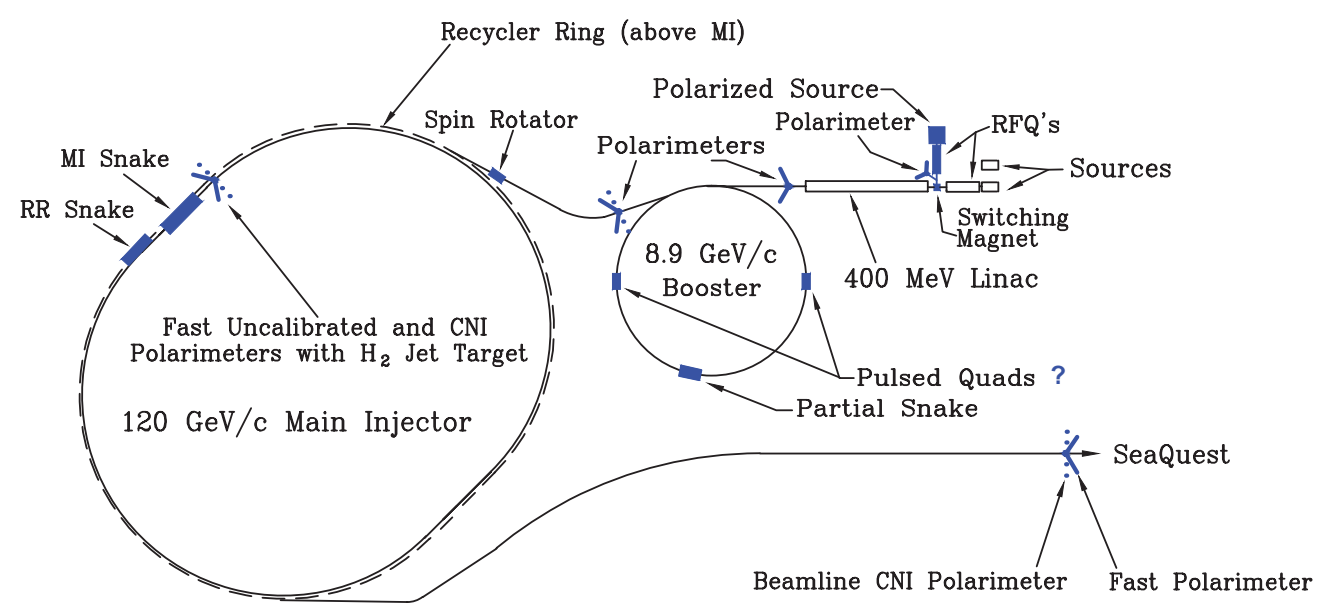

Figure 2: Major components needed (blue symbols) for polarized beam at Fermilab .

Preserving and controlling the polarization during acceleration and storage at energies up to hundreds of $\mathrm{GeV}$ is challenging due to many depolarizing resonances. In 1977 Derbenev and Kondratenko [12] proposed a way to overcome all depolarizing intrinsic resonances with arrangements of magnets now called Siberian snakes in which each snake rotates each beam particle's spin by $180^{\circ}$. This concept is currently used at BNL to achieve high-energy proton beams at RHIC, where polarizations of up to $65 \%$ for $100 \mathrm{GeV}$ beams and up to $60 \%$ for $255 \mathrm{GeV}$ beams have been reached.

Based on the current configurations at RHIC, the Spin@Fermi collaboration first recommended the use of two superconducting single-twist helical dipole Siberian snakes in the Main Injector. However, it was discovered in 2012 that there was no longer sufficient space in the Main Injector for two snakes at opposite locations around the ring. Back at the drawing board, Derbenev, Kondratenko, Courant, Krisch, some the of the world experts in Siberian snake design, and some undergraduate students came up with a novel solution: they found that a single, four-twist helical dipole Siberian snake with a dipole on each end [13] could be as efficient as a Siberian snake-pair to provide about $70 \%$ polarized beam to the SeaQuest spectrometer.

Accelerating particles to high energies, transverse magnetic fields in a snake are very effective in rotating the spin because of the near invariance of their $\int B \cdot d l$ under Lorentz boosts. However, the transverse magnetic fields cause orbit excursions inside the snake magnets; these excursions decrease with increasing energy. It was found that the beam excursions caused by a single helix were prohibitively large $(64 \mathrm{~mm})$ at the Main Injector injection energy of $8.9 \mathrm{GeV}$. Thus, focus shifted to a multi-twist helix. Multi-twist snakes with twist angles that are an even multiple of $\pi$ need only one vertical dipole at each end for optical transparency. An $8 \pi$ twist angle was demonstrated to reduce the orbit excursions greatly while maintaining an acceptable snake length [13].

Fig. 3 displays the spin components inside the four-twist helical snake, along with the magnetic field components and the horizontal and vertical excursions. The maximum transverse excursion inside the four-twist helical snake is $16.55 \mathrm{~mm}$ at the injection energy of $8.9 \mathrm{GeV}$. Thus the aperture 

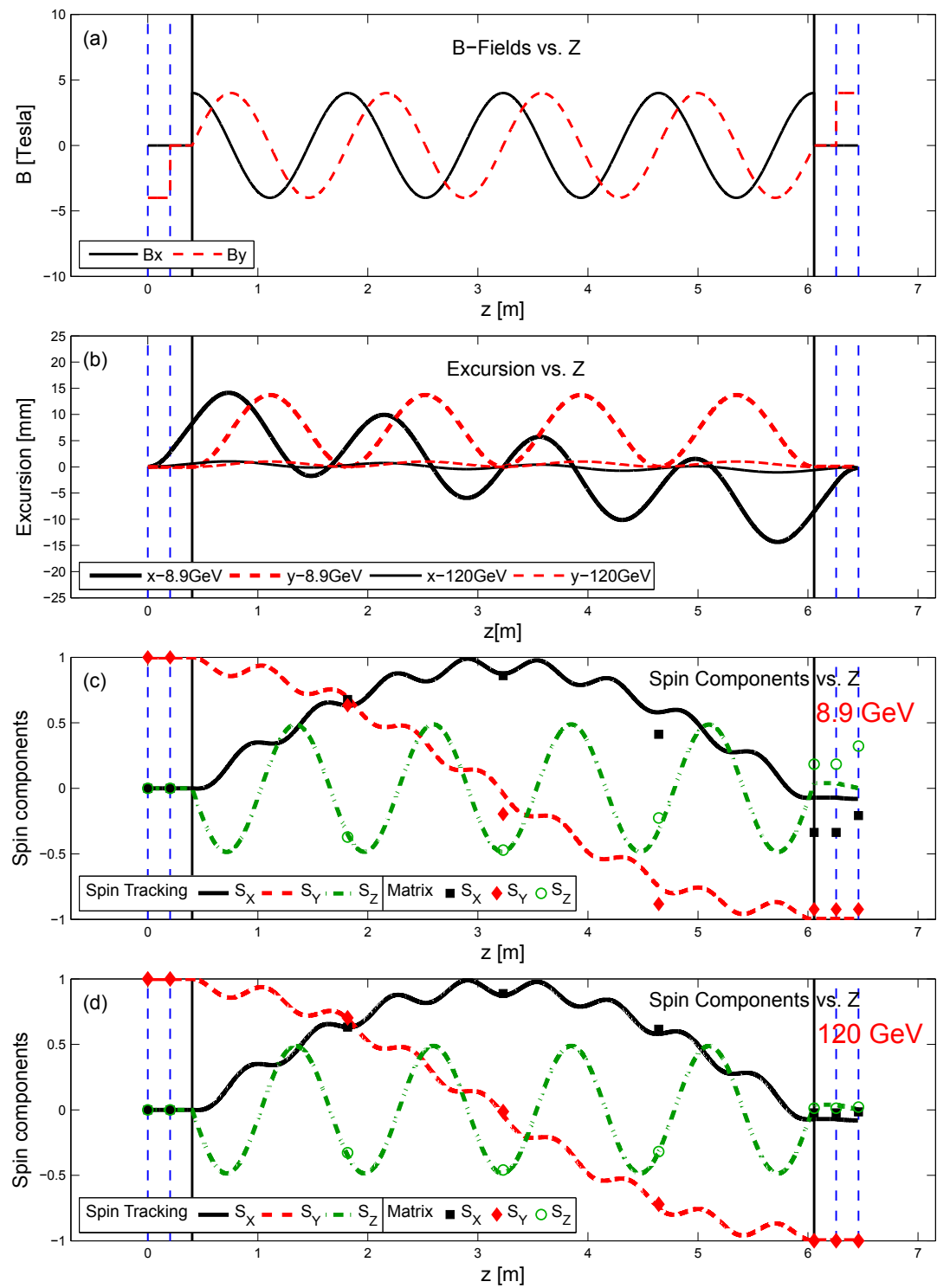

Figure 3: (a) Horizontal and vertical $B$-field components as a function of $z$ for a 4 T, 5.653 m, 4-twist helical dipole with 4 T, $0.203 \mathrm{~m}$ dipoles and $0.2 \mathrm{~m}$ gaps. (b) Horizontal and vertical orbit excursions as a function of $z$. (c) Radial, vertical, and longitudinal spin components as a function of $z$ for a beam energy of $8.9 \mathrm{GeV}$. (d) Spin components for a beam energy of $120 \mathrm{GeV}$.

required for a four-twist helix is nearly a factor of four smaller than that required by a single-twist helix, and the excursions fit into the existing beam pipe aperture.

Furthermore, recent studies by the Spin@Fermi Collaboration [13] have found that it may be possible to eliminate the $120 \mathrm{GeV}$ spin rotator in the extraction line that leads to the NM4 experimental area, if the Main Injector beam energy could be reduced from $120.0 \mathrm{GeV}$ to approximately $118.64 \mathrm{GeV}$. It was found that for a $G \gamma \approx 226.74$ the normal spin component at the last magnet 
before NM4 is near 99\%. Further studies with full spin tracking in the lattices for the Booster and the Main Injector, and the $2.4 \mathrm{~km}$ long extraction line are necessary to confirm this study.

\subsection{Full spin tracking in the Booster and Main Injector lattices}

While these results were considered promising enough to merit Stage-1 approval of E1027 by the Fermilab Program Advisory Committee and Fermilab Directorate in November 2012, in order to move forward, much more detailed and complete design studies are needed. The Spin@Fermi Collaboration has so far focused on the single-snake design for the Main Injector. Full spin tracking through the Main Injector as well as the Booster lattices will need to be performed. Lattice files from after the 2012-13 Main Injector upgrade have been obtained from the Fermilab Accelerator Division. Given that Fermilab uses version 8 of the Methodical Accelerator Design package [14] (MAD-8) from CERN and the BNL Collider-Accelerator Department uses version X (MAD-X) for lattice description and Zgoubi [15] for spin tracking, the lattices first needed to be translated to interact effectively with RHIC beam experts, such as Mei Bai from BNL. Particle transport (beta functions) have been verified against the original MAD-8 results, and preliminary intrinsic resonance strength calculations [17] have been performed so far, as shown in Fig. 4.

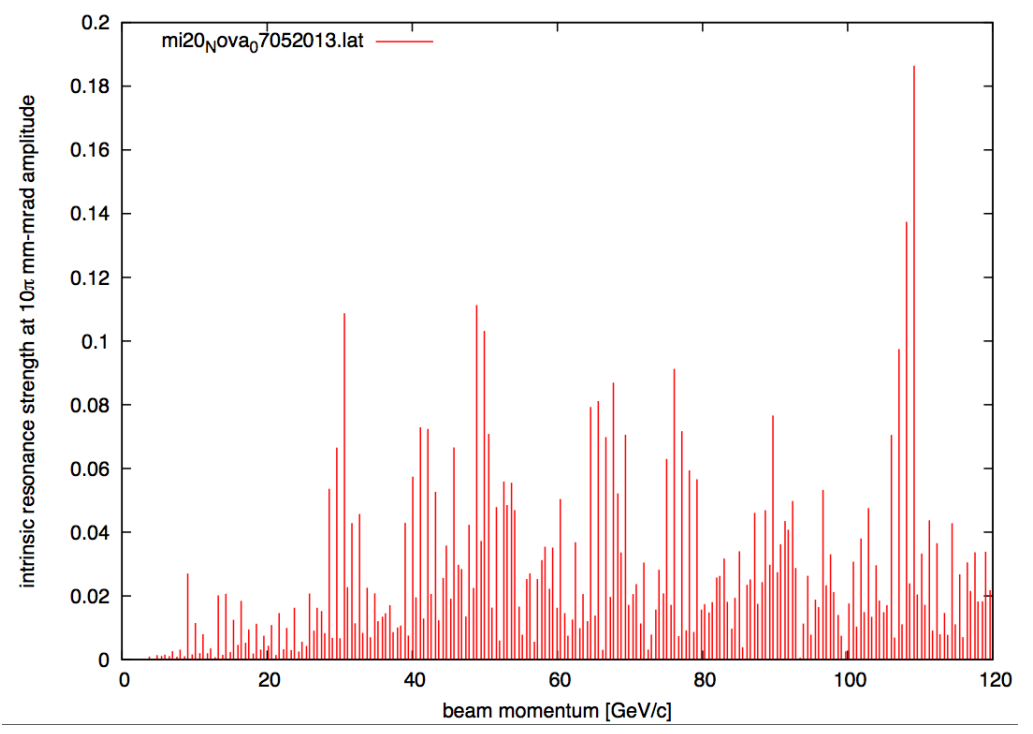

Figure 4: Intrinsic resonances at the Main Injector assuming $10 \pi \mathrm{mm}$-mrad emittance (using post intensityupgrade lattice).

Spin tracking for a single particle will have to be set up next for the Main Injector and Booster lattices with the currently proposed snake configurations implemented. Once single-particle spin tracking is understood, the next step will be to set up multi-particle spin tracking at different emittances, and then to add errors such as orbit errors and quadrupole misalignment and rolls. Once completed, systematic spin tracking calculations need to be performed, where tolerances on beam emittance and tolerances on various imperfections, such as orbit and snake imperfections, will have to be studied. 
Earlier calculations performed by the Spin@Fermi collaboration [16, 8] on pre Main Injector intensity-upgrade lattices have indicated that all depolarizing resonance strengths were below 0.2 for a single particle at $10 \pi \mathrm{mm}$-mrad betatron amplitude up to $120 \mathrm{GeV}$. This indicates ${ }^{1}$ that a single snake may be sufficient to overcome all depolarizing resonances and maintain polarization for $120 \mathrm{GeV}$ beams. The expected strengths of the intrinsic and imperfection depolarizing resonances in the Main Injector will need to be re-evaluated based on post intensity-upgrade lattices.

\section{Summary}

The combination of high luminosity, large $x$-coverage and a high-intensity polarized beam makes Fermilab arguably the best place to measure single-spin asymmetries in polarized Drell-Yan scattering with high precision. It would allow the first measurement of the sign, the magnitude, and the shape of the Sivers function with sufficient precision to verify this fundamental prediction of QCD conclusively. Current estimates suggest that for $\$ 10 \mathrm{M}$ a $150 \mathrm{nA}$ proton beam with $70 \%$ polarization could be available after the end of data collection for SeaQuest.

\section{References}

[1] D.W. Sivers, Phys. Rev. D41, 83 (1990); D43, 261 (1991).

[2] J.C. Collins, Phys. Lett. B536, 43 (2002).

[3] S.J. Brodsky et al., Nucl. Phys. B642, 344 (2002).

[4] (HERMES Collaboration) A. Airapetian et al., Phys. Rev. Lett. 94, 012002 (2005).

[5] (COMPASS Collaboration) V.Y. Alexakhin et al., Phys. Rev. Lett. 94, 202002 (2005).

[6] M. Anselmino. et al., Eur. Phys. Jour. A39, 89 (2009).

[7] Z. Lu, B.Q. Ma. and J. Zhu, [hep-ph/1108 . 4974].

[8] (Spin@ Fermi Collaboration) E.D. Courant, et al., Updated Report Acceleration of Polarized Protons to 120-150 GeV/c at Fermilab, [physics . acc-ph/1110.3042].

[9] A. Bacchetta and M. Radici, [hep-ph/1107.5755].

[10] M. Anselmino et al., Phys. Rev. D79, 054010 (2009); plus private communications.

[11] http://conferences.fnal.gov/polarizedprotons/

[12] Y.S. Derbenev and A.M. Kondratenko, in proceedings of $10^{\text {th }}$ Int. Conf. on High Energy Accel., Protvino, 70 (1977).

[13] F. Antoulinakis et al., [physics.acc-ph/1309.1063].

[14] http://mad.web.cern.ch/mad/

[15] http://sourceforge.net/projects/zgoubi/

[16] V.A Anferov et al., Department of Physics, University of Michigan, UM-HE-95-09 (July 1995).

[17] Mei Bai, private communications.

\footnotetext{
${ }^{1}$ According to a rule of thumb by E.D. Courant, the number of Siberian snakes needed to overcome all depolarizing resonances is at least five times the largest depolarizing resonance strength. This ensures that the snake dominates the spin motion in the ring.
} 